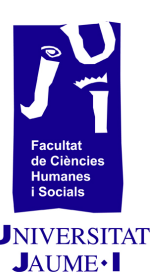

\title{
Análisis de la transparencia financiera en la web de los clubes de la Liga de Fútbol Profesional
}




\section{Justificación de la temática}

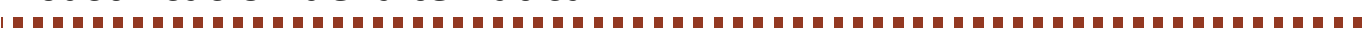

En los últimos años, el fútbol se ha convertido en una de las industrias económicas más importantes del deporte universal. Lo que en los años cincuenta y sesenta era un juego local, en las décadas posteriores comenzó a erigirse como uno de los principales pasatiempos de la gente, sobre todo en España. Sin embargo, fue a partir de los años ochenta cuando el fútbol se convirtió en una industria global. Un buen ejemplo de ello es la comparación entre la facturación del FC Barcelona en la temporada 1978-1979, en la que marcó unas cifras de 1000 millones de pesetas ( 6 millones de euros actuales), y la alcanzada en la 2014-2015: 561 millones de euros.

De este modo, no es extraño que actualmente el fútbol profesional en España genere más de 140000 empleos a tiempo completo y un valor añadido bruto (VAB) de más de 7600 millones de euros, casi un $1 \%$ del PIB español, según el estudio "Impacto socio-económico del fútbol profesional en España», elaborado por KPMG.

Sin embargo, en el mundo del deporte rey, tras diferentes polémicas económicas, especialmente en torno a los precios finales de los fichajes, la transparencia económica de los distintos clubes se ha convertido en una de las cuestiones más analizadas. El aumento de las capacidades de monitorización y fiscalización de las actividades de las empresas por parte del público ha supuesto que la transparencia se configure como un pilar fundamental de credibilidad.

Fruto de ello, los máximos organismos del fútbol han implementado acciones para evaluar el grado de transparencia de los clubes profesionales y propiciar el aumento de la información que estos ofrecen a los aficionados y a la sociedad en general. Por ello, resulta interesante y necesario evaluar la situación de la transparencia económica en el fútbol español y determinar hasta qué punto este apartado económico ha irrumpido en la realidad en los principales clubes de LaLiga.

\section{Objetivos}

1. Determinar la trascendencia del apartado financiero en los equipos de fútbol profesionales.

2. Comprobar si la web de los clubes de fútbol profesionales representa la herramienta principal en el apartado de comunicación financiera. 


\section{Paso de un juego de entretenimiento a un mercado global}

En la actualidad, el fútbol es el deporte más universal y supone una multitud de articulaciones sociales (Antezana, 2003). Según Bromberger (1992), la popularidad de los deportes radica, en gran medida, en su capacidad de encarnar el ideal de las sociedades democráticas. El fútbol, concretamente, es uno de los espectáculos deportivos seguidos con mayor pasión por parte de todos los ciudadanos del mundo (Bromberger, 2001; González, 2011).

Sin embargo, con el paso de los años, este espectáculo popular de antaño se ha convertido en una gran industria de carácter global y un modelo de negocio que arrastra miles de millones, en el que fluctúan numerosos intereses. Un ejemplo de ello podemos encontrarlo en el FC Barcelona, uno de los clubes españoles más laureados en el panorama futbolístico mundial, ya que si en la temporada 1978-1979 su facturación se tasaba en 1000 millones de pesetas (6 millones de euros actuales), en la 2014-2015 esta cifra ascendía hasta los 561 millones (Gay, 2016).

Es en los años ochenta y noventa cuando se comienzan a dar los primeros pasos hacia la industria económica que hoy engloba el fútbol. Las ingentes deudas de los clubes obligaron al Estado a tomar medidas y las extintas formas jurídicas de clubes deportivos fueron transformadas en Sociedades Anónimas Deportivas (Gutiérrez Gilsanz, 2001). Una de las principales razones de esta decisión radicó en el hecho de que la personificación jurídica de club no estaba preparada para operar en el mercado y manejar grandes cantidades monetarias como las que el fútbol estaba comenzando a generar.

\section{Importancia de la página web en la comunicación financiera}

A la vez que se produce el proceso de profesionalización de los clubes de fútbol antes explicado, se lleva a cabo otra evolución que también marcará un antes y un después en el apartado comunicativo de las empresas, un proceso que engloba el apartado financiero. En la segunda mitad del siglo $\mathrm{xx}$, las sociedades industrializadas dan el salto definitivo hacia un nivel de vida más elevado (hecho que tiene como consecuencia un aumento del nivel de instrucción de la sociedad). En otras palabras, este cambio de modelo comporta una "subida de nivel» y las organizaciones tienen que enfrentarse a un ambiente más exigente y complejo, donde también están obligadas a realizar un mayor esfuerzo financiero en cuanto a comunicación (Piazzo, 2012).

Antes de introducir el elemento en línea en este contexto de necesidad comunicativa, conviene hacer una distinción. Mientras que en una empresa al uso los objetivos de la comunicación 
financiera serían aumentar los públicos de referencia, aportar confianza sobre la solidez económica de la organización e informar de las necesidades legales de estas entidades con los inversores (Pérez-Díaz y López, 2003), el carácter pasional que rodea al mundo del fútbol cambia la percepción que estas sociedades publiquen en su apartado financiero, y es que la percepción prácticamente visceral de estas organizaciones por parte de sus aficionados todavía aumenta la necesidad de transparencia antes justificada generalmente.

Al introducir el marco en línea en la ecuación, es innegable que esta herramienta no solo ha facilitado las posibilidades que tiene una organización de poder realizar su comunicación externa, sino que las ha aumentado. En el apartado estrictamente financiero, existe una vertiente generalizada de liderazgo en las organizaciones que sí han comunicado su informe anual en la web, un dato reforzado por el hecho de que las empresas españolas con mayor reputación y mejor imagen son también las que refuerzan su actividad comunicativa incluyendo acciones en pro de la transparencia en web (Estrada, 2012).

Este mismo estudio hace referencia a una diferenciación entre la gestión de transparencia financiera que hacen las empresas en función de si cotizan o no en bolsa. En él se explica que las empresas que no tienen esta obligación no publican su informe anual. Esta tendencia se explica como errónea, ya que esta acción representa en sí misma una oportunidad para favorecer su transparencia y su reputación. En el estudio posterior, se analizarán tanto clubes que coticen en bolsa (con la obligación de hacer públicas sus cuentas) como equipos que no tengan esta obligación, con lo que también se obtendrán conclusiones sobre si este aprovechamiento de reputación financiera se está llevando a cabo en la élite del fútbol español, en este caso en clubes como el Real Madrid que no son Sociedades Anónimas Deportivas.

Llegados a este punto, conviene delimitar el marco al que se enfrentan los clubes de fútbol profesional respecto al grado de transparencia financiera que tienen que cubrir en su web. Más allá de las obligaciones de transparencia financiera que tienen estas entidades en el ámbito general, la Ley de Transparencia aprobada en el año 2013 y que entró en vigor el 10 de diciembre de 2014 determina un cambio fundamental: se obliga a los clubes a ofrecer información financiera y contable de las entidades deportivas en sus respectivas páginas web debido a su relevancia pública (Riesgo, 2016).

Esta información que los clubes deben proporcionar se divide en dos apartados: el institucional, organizativo y de planificación, y el económico, presupuestario y estadístico. Concretamente, en la página web de cada club debe aparecer una sección específica, o 
bien un portal de transparencia, que ofrezca la información requerida rigiéndose por los requisitos siguientes:

- Accesibilidad: documentación bien organizada que permita una mayor facilidad de búsqueda de los interesados.

- Interoperabilidad: que ofrezca la posibilidad de compartir y operar la documentación ofrecida.

- Reutilización: el formato tiene que permitir que la información pueda reutilizarse tantas veces como se necesite.

\section{Muestra escogida. Justificación}

La muestra (10 clubes de fútbol de la primera división española), además de englobar un porcentaje importante de los equipos de la primera división de la Liga de Fútbol Profesional, engloba dos tipos de clubes: Sociedades Anónimas Deportivas y Asociaciones Deportivas no Mercantiles. Esta dualidad permitirá establecer las diferencias entre ambos modelos en lo que respecta a su transparencia financiera en la web.

La muestra escogida también representa, en su mayoría, a la élite del fútbol español. Es decir, los resultados obtenidos mostrarán el grado de transparencia financiera de la élite del deporte mayoritario en España.

Por último, haber incluido clubes valencianos en esta muestra nos permite, por cercanía, poder profundizar más sobre las razones que llevan a estos clubes a publicar estos documentos financieros y cómo se organizan exactamente.

\section{Metodología}

El análisis se va a llevar a cabo a través de las siguientes variables extraídas del documento Índice de Transparencia de los Clubes de Fútbol (INFUT), elaborado por Transparency International España:

\section{¿Facilitan los clubes la siguiente información en su web?}

1. Publicación de las cuentas anuales

2. Incorporación de un portal (o sección específica) de transparencia

3. Publicación de informes externos de auditoría sobre las cuentas del club

4. Fecha de entrega al Consejo Superior de Deportes de las últimas cuentas anuales

5. Fecha de depósito en el Registro Mercantil de las últimas cuentas anuales 
6. Fecha de envío a la Liga de Fútbol Profesional de las últimas cuentas anuales

7. Publicación de los estados financieros intermedios individuales y consolidados

8. Publicación del punto de equilibrio del club

9. Publicación de las cuentas anuales de entidades vinculadas

10. Ratio de autonomía financiera: patrimonio neto/(pasivo + patrimonio neto)

11. Publicación de retribuciones totales de la primera plantilla/presupuesto

12. Ratio deudas del club/ingresos relevantes

13. Ratio del importe invertido primera plantilla/ingresos relevantes de la última temporada

14. Desglose de los ingresos relevantes del club

15. Detalle de los gastos relevantes del club

16. Detalle de gastos asociados a la primera plantilla

17. Desglose de endeudamiento del club

18. Listado de deudas con las administraciones públicas

19. Listado de deudas y créditos con los empleados

20. Listado de deudas y créditos con el personal deportivo

21. Publicación del importe recibido por traspasos e importe de fichajes realizados en las dos últimas temporadas

22. Publicación del tope salarial del club

23. Publicación de las ayudas públicas y subvenciones recibidas

24. Publicación de los presupuestos

\section{Resultados del análisis}

Pese a detallar los resultados obtenidos en la fase de análisis de forma individualizada, en ningún momento se va a desvelar la identidad real del club, que será identificado en cada caso con una letra. Además, la primera valoración numérica de cada equipo corresponde con la cantidad de variables que el club presenta del total de 24 que se acaban de nombrar al explicar la metodología.

CLUB A: (17/24). Una puntuación que, como más tarde se podrá comprobar, representa la media de resultados obtenidos. Más allá de la presencia o ausencia de los elementos analizados, es destacable que una considerable parte de ellos se facilitan en el apartado de transparencia de la página web. 


\begin{tabular}{|c|c|}
\hline CUENTAS ANUALES & I \\
\hline PORTAL DE TRANSPARENCIA & I \\
\hline INFORMES EXTERNOS AUDITORÍA & I \\
\hline ENTREGACUENTASANUALES AL CSD & I \\
\hline $\begin{array}{l}\text { FECHADE ENTREGA EN EL REG. MERCANTIL } \\
\end{array}$ & 0 (no es sociedad mercantil) \\
\hline ENVIO DE LAS CUENTAS A LA LIGA & $\mathrm{I}$ \\
\hline PUBLICACIÓN DE LOS ESTADOS FINANC. & I \\
\hline PUBLICACIÓN PUNTO DE EQUILIBRIO & I \\
\hline CUENTAS ANUALES DE ENT. VINCULADAS & 0 \\
\hline RATIODEAUTONOMÍA FINANCIERA & 0 \\
\hline RETRIBUCIONES1ä PLANTILLA/PRESUPUESTO & 0 \\
\hline RATIO DEUDAS/INGRESOS RELEVANTES & I \\
\hline INVERSIÓN 1äPLANTILLA/ING. REL. (último año) & I \\
\hline DESGLOSE INGRESOS RELEVANTES & I \\
\hline DETALLE GASTOS RELEVANTES & I \\
\hline DETALLE GASTOS DE 19 PLANTILLA & 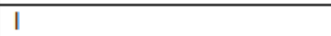 \\
\hline DESGLOSE DE ENDEUDAMIENTO & I \\
\hline LISTA DEUDAS CON ADMIN. PÚBLICAS & I \\
\hline DEUDASY CRÉDITOSCON LOS EMPLEADOS & 0 \\
\hline DEUDASY CRÉDITOSCON EL PERSONAL DEP. & 0 \\
\hline IMPORTE DE TRASPASOS Y FICHAJES (2 últ años) & 0 \\
\hline TOPE SALARIAL DEL CLUB & I \\
\hline AYUDAS PÚBLICAS Y SUBVENCIONES RECIBIDAS & I \\
\hline PUBLICACIÓN DE LOS PRESUPUESTOS & I \\
\hline
\end{tabular}

CLUB B: (24/24). Transparencia total. El club facilita todos los datos analizados $y$, además, utiliza exactamente la misma estructura que la que se ha utilizado para hacer el análisis INFUT. Esta influencia es evidente, ya que incluso se mantiene el orden de este barómetro de medición en la web del equipo. Se han basado en el INFUT para lograr un elevado grado de transparencia.

\begin{tabular}{|c|c|}
\hline CUENTASANUALES & $\mathrm{I}$ \\
\hline PORTAL DE TRANSPARENCIA & $\mathrm{I}$ \\
\hline INFORMES EXTERNOS AUDITORÍA & $\mathrm{I}$ \\
\hline ENTREGACUENTASANUALES AL CSD & $\mathrm{I}$ \\
\hline FECHADE ENTREGA EN EL REG. MERCANTIL & $\mathrm{I}$ \\
\hline ENVIO DE LAS CUENTAS A LA LIGA & $\mathrm{I}$ \\
\hline $\begin{array}{l}\text { PUBLICACIÓN DE LOS ESTADOS FINANC. } \\
\text { PINT }\end{array}$ & $\mathrm{I}$ \\
\hline PUBLICACIÓN PUNTO DE EQUILIBRIO & $\mathrm{I}$ \\
\hline CUENTAS ANUALES DE ENT. VINCULADAS & $\mathrm{I}$ \\
\hline RATIDDEAUTONOMÍA FINANCIERA & $\mathrm{I}$ \\
\hline RETRIBUCIONES 1ą PLANTILLA/PRESUPUESTO & $\mathrm{I}$ \\
\hline RATIO DEUDAS/INGRESOS RELEVANTES & $\mathrm{I}$ \\
\hline INVERSIÓN 1ạPLANTILLA/ING. REL. (último año) & $\mathrm{I}$ \\
\hline DESGLOSE INGRESOS RELEVANTES & $\mathrm{I}$ \\
\hline DETALLE GASTOS RELEVANTES & $\mathrm{I}$ \\
\hline $\begin{array}{l}\text { DETALLE GASTOS DE 1a PLANTILLA } \\
\end{array}$ & $\mathrm{I}$ \\
\hline DESGLOSE DEENDEUDAMIENTO & $\mathrm{I}$ \\
\hline LISTA DEUDAS CON ADMIN. PÚBLICAS & $\mathrm{I}$ \\
\hline DEUDASY CRÉDITOSCON LOS EMPLEADOS & $\mathrm{I}$ \\
\hline DEUDASY CRÉDITOSCON EL PERSONAL DEP. & $\mathrm{I}$ \\
\hline IMPORTE DE TRASPASOS Y FICHAIES (2 últ años) & $\mathrm{I}$ \\
\hline TOPE SALARIAL DEL CLUB & $\mathrm{I}$ \\
\hline AYUDAS PÚBLICAS Y SUBVENCIONES RECIBIDAS & $\mathrm{I}$ \\
\hline PUBLICACIÓN DE LOS PRESUPUESTOS & $\mathrm{I}$ \\
\hline
\end{tabular}

CLUB C: (17/24). La puntuación lograda se repite respecto al primer caso. Pese a que no se detecta una influencia directa al INFUT como el caso anterior (ni siquiera nombra al baremo), sí que se observan similitudes, ya que muchos apartados del portal de 
transparencia tienen exactamente la misma nomenclatura que la que reciben en el INFUT. De nuevo, la accesibilidad sigue siendo buena en este caso, ya que para acceder a gran parte de la información ni siquiera hay que abrir ningún documento.

\begin{tabular}{|c|c|}
\hline CUENTASANUALES & | I \\
\hline PORTALDE TRANSPARENCIA & I \\
\hline INFORMES EXTERNOS AUDITORÍA & I \\
\hline ENTREGACUENTAS ANUALES AL CSD & I \\
\hline FECHADE ENTREGA EN EL REG. MERCANTIL & I \\
\hline ENVIO DE LAS CUENTAS A LA LIGA & I \\
\hline PUBLICACIÓN DE LOS ESTADOS FINANC. & I \\
\hline PUBLICACIÓN PUNTO DE EQUILIBRIO & I \\
\hline CUENTAS ANUALES DE ENT. VINCULADAS & 0 \\
\hline RATIODEAUTONOMÍA FINANCIERA & I \\
\hline RETRIBUCIONES 1a PLANTILLA/PRESUPUESTO & 0 \\
\hline RATIO DEUDAS/INGRESOS RELEVANTES & 0 \\
\hline INVERSIÓN 1ạPLANTILLA/ING. REL. (último año) & I \\
\hline DESGLOSE INGRESOS RELEVANTES & I \\
\hline DETALLE GASTOS RELEVANTES & I \\
\hline DETALLE GASTOS DE 1ạ PLANTILLA & I \\
\hline DESGLOSE DE ENDEUDAMIENTO & I \\
\hline LISTA DEUDAS CON ADMIN. PÚBLICAS & I \\
\hline DEUDASY CRÉDITOSCON LOS EMPLEADOS & 0 \\
\hline DEUDASY CRÉDITOSCON EL PERSONAL DEP. & 0 \\
\hline IMPORTE DE TRASPASOS Y FICHAJES (2 últ años) & 0 \\
\hline TOPE SALARIAL DEL CLUB & 0 \\
\hline AYUDAS PÚBLICASY SUBVENCIONES RECIBIDAS & 1 \\
\hline PUBLICACIÓN DE LOS PRESUPUESTOS & $\mathrm{I}$ \\
\hline
\end{tabular}

CLUB D: (24/24). De nuevo, transparencia total. El análisis es exactamente el mismo al del club B. El baremo utilizado prácticamente se traslada al apartado de transparencia de la web del equipo y para acceder a este tipo de información, no hace falta entrar a ningún documento.

\begin{tabular}{|c|c|}
\hline CUENTASANUALES & $\mathrm{I}$ \\
\hline PORTALDE TRANSPARENCIA & $\mathrm{I}$ \\
\hline INFORMES EXTERNOS AUDITORÍA & $\mathrm{I}$ \\
\hline ENTREGACUENTAS ANUALES AL CSD & $\mathrm{I}$ \\
\hline FECHADE ENTREGA EN EL REG. MERCANTIL & $\mathrm{I}$ \\
\hline ENVIO DE LAS CUENTAS A LA LIGA & $\mathrm{I}$ \\
\hline PUBLICACIÓN DE LOS ESTADOS FINANC. & $\mathrm{I}$ \\
\hline PUBLICACIÓN PUNTO DE EQUILIBRIO & $\mathrm{I}$ \\
\hline CUENTASANUALES DE ENT. VINCULADAS & $\mathrm{I}$ \\
\hline RATIO DEAUTONOMÍA FINANCIERA & $\mathrm{I}$ \\
\hline RETRIBUCIONES 1@ PLANTILLA/PRESUPUESTO & $\mathrm{I}$ \\
\hline RATIO DEUDAS/INGRESOS RELEVANTES & $\mathrm{I}$ \\
\hline INVERSIÓN 1aPLANTILLA/ING. REL. (último año) & $\mathrm{I}$ \\
\hline DESGLOSE INGRESOS RELEVANTES & $\mathrm{I}$ \\
\hline DETALLE GASTOS RELEVANTES & $\mathrm{I}$ \\
\hline DETALLE GASTOS DE 1@ PLANTILLA & $\mathrm{I}$ \\
\hline DESGLOSE DE ENDEUDAMIENTO & $\mathrm{I}$ \\
\hline LISTA DEUDAS CON ADMIN. PÚBLICAS & $\mathrm{I}$ \\
\hline DEUDASY CRÉDITOSCON LOS EMPLEADOS & $\mathrm{I}$ \\
\hline DEUDASY CRÉDITOSCON EL PERSONAL DEP. & $\mathrm{I}$ \\
\hline IMPORTE DE TRASPASOS Y FICHAJES (2 últ años) & $\mathrm{I}$ \\
\hline TOPE SALARIAL DEL CLUB & $\pi$ \\
\hline AYUDAS PÚBLICAS Y SUBVENCIONES RECIBIDAS & $\mathrm{I}$ \\
\hline PUBLICACIÓN DE LOS PRESUPUESTOS & I \\
\hline
\end{tabular}


CLUB E: (18/24). Se observa que la puntuación se va homogeneizando. Presencia notable de las variables analizadas. Sin embargo, con este equipo se empieza a ver una tendencia que se va a repetir en algunos casos: el apartado de transparencia de la web es prácticamente anecdótico, ya que las 18 variables aportadas por el club están situadas en dos archivos, además escaneados, lo que dificulta mucho la accesibilidad.

\begin{tabular}{|c|c|}
\hline CUENTASANUALES & I \\
\hline PORTAL DE TRANSPARENCIA & I \\
\hline INFORMES EXTERNOS AUDITORÍA & I \\
\hline ENTREGA CUENTASANUALES AL CSD & 0 \\
\hline FECHADE ENTREGA EN EL REG. MERCANTIL & I \\
\hline ENVIO DE LAS CUENTAS A LA LIGA & 0 \\
\hline PUBLICACIÓN DE LOS ESTADOS FINANC. & I \\
\hline PUBLICACIÓN PUNTO DE EQUILIBRIO & $\mathrm{I}$ \\
\hline CUENTAS ANUALES DE ENT. VINCULADAS & 0 \\
\hline RATIO DE AUTONOMÍA FINANCIERA & I \\
\hline RETRIBUCIONES 1a PLANTILLA/PRESUPUESTO & $\mathrm{I}$ \\
\hline RATIO DEUDAS/INGRESOS RELEVANTES & I \\
\hline INVERSIÓN 1aPLANTILLA/ING. REL. (último año) & 0 \\
\hline DESGLOSE INGRESOS RELEVANTES & I \\
\hline DETALLE GASTOS RELEVANTES & I \\
\hline DETALLE GASTOS DE 1a PLANTILLA & I \\
\hline DESGLOSE DE ENDEUDAMIENTO & I \\
\hline LISTA DEUDAS CON ADMIN. PÚBLICAS & 0 \\
\hline DEUDASY CRÉDITOSCON LOS EMPLEADOS & $\mathrm{I}$ \\
\hline DEUDASY CRÉDITOSCON EL PERSONAL DEP. & I \\
\hline IMPORTE DE TRASPASOS Y FICHAJES (2 últ años) & 0 \\
\hline TOPE SALARIAL DEL CLUB & $\mathrm{I}$ \\
\hline AYUDASPÚBLICASY SUBVENCIONES RECIBIDAS & I \\
\hline PUBLICACIÓN DE LOS PRESUPUESTOS & $\mathrm{I}$ \\
\hline
\end{tabular}

CLUB F: (21/24). Muy buena puntuación que se suma a una muy buena accesibilidad. En este caso, los estatutos están informatizados, por lo que muchos de los datos analizados se pueden revisar con bastante facilidad. Además, la presencia de un índice con un lenguaje sencillo en la sección de transparencia de la web también facilita el trabajo. 


\begin{tabular}{|c|c|}
\hline CUENTASANUALES & $\mathrm{I}$ \\
\hline PORTAL DE TRANSPARENCIA & $\mathrm{I}$ \\
\hline INFORMES EXTERNOS AUDITORÍA & $\mathrm{I}$ \\
\hline ENTREGACUENTAS ANUALES AL CSD & $\mathrm{I}$ \\
\hline FECHADE ENTREGA EN EL REG. MERCANTIL & $\mathrm{I}$ \\
\hline ENVIO DE LAS CUENTAS A LA LIGA & $\mathrm{I}$ \\
\hline PUBLICACIÓN DE LOS ESTADOS FINANC. & 0 \\
\hline PUBLICACIÓN PUNTO DE EQUILIBRIO & $\mathrm{I}$ \\
\hline CUENTAS ANUALES DE ENT. VINCULADAS & 0 \\
\hline RATIODEAUTONOMÍA FINANCIERA & 0 \\
\hline RETRIBUCIONES 1@a PLANTILLA/PRESUPUESTO & $\mathrm{I}$ \\
\hline RATIO DEUDAS/INGRESOS RELEVANTES & $\mathrm{I}$ \\
\hline INVERSIÓN 1ạPLANTILLA/ING. REL. (último año) & $\mathrm{I}$ \\
\hline DESGLOSE INGRESOS RELEVANTES & $\mathrm{I}$ \\
\hline DETALLE GASTOS RELEVANTES & $\mathrm{I}$ \\
\hline DETALLE GASTOS DE 1 PLANTILLA & $\mathrm{I}$ \\
\hline DESGLOSE DE ENDEUDAMIENTO & $\mathrm{I}$ \\
\hline LISTA DEUDAS CON ADMIN. PÚBLICAS & $\mathrm{I}$ \\
\hline DEUDASY CRÉDITOS CON LOS EMPLEADOS & $\mathrm{I}$ \\
\hline DEUDASY CRÉDITOSCON EL PERSONAL DEP. & $\mathrm{I}$ \\
\hline IMPORTE DE TRASPASOS Y FICHAJES (2 últ años) & $\mathrm{I}$ \\
\hline TOPE SALARIAL DEL CLUB & $\mathrm{I}$ \\
\hline AYUDAS PÚBLICAS Y SUBVENCIONES RECIBIDAS & $\mathrm{I}$ \\
\hline PUBLICACIÓN DE LOS PRESUPUESTOS & $\mathrm{I}$ \\
\hline
\end{tabular}

CLUB G: (15/24). Es el segundo club con peor puntuación de todos los analizados. Pero aunque pueda parecer contradictorio, es el club que más peso le da al apartado de transparencia de su web. Facilita bastante información (tanto del baremo utilizado en este análisis como ajena). Además, todos los indicadores que contesta, a excepción de tres, se pueden consultar sin la necesidad de mirar en documentos, están presentes en la web.

\begin{tabular}{|c|c|}
\hline CUENTASANUALES & 1 \\
\hline PORTAL DE TRANSPARENCIA & $\mathrm{I}$ \\
\hline INFORMES EXTERNOS AUDITORÍA & 0 \\
\hline ENTREGACUENTASANUALES AL CSD & $\mathrm{I}$ \\
\hline FECHA DE ENTREGA EN EL REG. MERCANTIL & $\mathrm{I}$ \\
\hline ENVIO DE LAS CUENTAS A LA LIGA & $\mathrm{I}$ \\
\hline PUBLICACIÓN DE LOS ESTADOS FINANC. & 0 \\
\hline PUBLICACIÓN PUNTO DE EQUILIBRIO & 1 \\
\hline CUENTASANUALES DE ENT. VINCULADAS & 0 \\
\hline RATIO DEAUTONOMÍA FINANCIERA & I \\
\hline RETRIBUCIONES 1@ PLANTILLA/PRESUPUESTO & $\mathrm{I}$ \\
\hline RATIO DEUDAS/INGRESOS RELEVANTES & $\mathrm{I}$ \\
\hline INVERSIÓN 1ạPLANTILLA/ING. REL. (último año) & $\mathrm{I}$ \\
\hline DESGLOSE INGRESOS RELEVANTES & I \\
\hline DETALLE GASTOS RELEVANTES & $\mathrm{I}$ \\
\hline DETALLE GASTOS DE 1a PLANTILLA & $\mathrm{I}$ \\
\hline DESGLOSE DE ENDEUDAMIENTO & $\mathrm{I}$ \\
\hline LISTA DEUDAS CON ADMIN. PÚBLICAS & 0 \\
\hline DEUDASY CRÉDITOSCON LOS EMPLEADOS & 0 \\
\hline DEUDASY CRÉDITOSCON EL PERSONAL DEP. & 0 \\
\hline IMPORTE DE TRASPASOS Y FICHAJES (2 últ años) & 0 \\
\hline TOPE SALARIAL DEL CLUB & 0 \\
\hline AYUDAS PÚBLICAS Y SUBVENCIONES RECIBIDAS & 0 \\
\hline PUBLICACIÓN DE LOS PRESUPUESTOS & 1 \\
\hline
\end{tabular}

CLUB H: (14/24). Se trata del equipo con peor resultado. Además, es el único club de los 10 analizados que no cuenta con un apartado de transparencia en su web. Respecto a las variables 
facilitadas, para acceder a ellas hay que leer un archivo escaneado que dificulta la consulta de cualquier dato concreto.

\begin{tabular}{|c|c|}
\hline CUENTAS ANUALES & I \\
\hline PORTAL DE TRANSPARENCIA & 0 \\
\hline INFORMES EXTERNOS AUDITORÍA & I \\
\hline ENTREGACUENTAS ANUALES AL CSD & 0 \\
\hline FECHA DE ENTREGA EN EL REG. MERCANTIL & 0 \\
\hline ENVIO DE LAS CUENTAS A LA LIGA & 0 \\
\hline PUBLICACIÓN DE LOS ESTADOS FINANC. & 0 \\
\hline PUBLICACIÓN PUNTO DE EQUILIBRIO & I \\
\hline CUENTAS ANUALES DE ENT. VINCULADAS & I \\
\hline RATIODEAUTONOMÍA FINANCIERA & I \\
\hline RETRIBUCIONES 1ä PLANTILLA/PRESUPUESTO & I \\
\hline RATIO DEUDAS/INGRESOS RELEVANTES & I \\
\hline INVERSIÓN 1ạLANTILLA/ING. REL. (último año) & I \\
\hline DESGLOSE INGRESOS RELEVANTES & I \\
\hline DETALLE GASTOS RELEVANTES & I \\
\hline DETALLE GASTOS DE 1’ PLANTILLA & I \\
\hline DESGLOSE DE ENDEUDAMIENTO & I \\
\hline LISTA DEUDAS CON ADMIN. PÚBLICAS & 0 \\
\hline DEUDASY CRÉDITOS CON LOS EMPLEADOS & 1 \\
\hline DEUDASY CRÉDITOS CON EL PERSONAL DEP. & 0 \\
\hline IMPORTE DE TRASPASOS Y FICHAJES (2 últ años) & 0 \\
\hline TOPE SALARIAL DEL CLUB & 0 \\
\hline AYUDAS PÚBLICAS Y SUBVENCIONES RECIBIDAS & 0 \\
\hline PUBLICACIÓN DE LOS PRESUPUESTOS & I \\
\hline
\end{tabular}

CLUB I: (21/24). De nuevo muy buena puntuación, rozando la perfección. Un buen dato al cual se suma una buena accesibilidad. Únicamente basta con leer el índice que hay en el apartado de transparencia y ya se sabe dónde acudir para acceder a las variables que se han analizado. Por otra parte, esta facilidad a determinados datos presentes en el INFUT también evidencian la influencia que ha tenido este barómetro en la transparencia de este equipo.

\begin{tabular}{|l|l|}
\hline CUENTASANUALES & $\mathrm{I}$ \\
\hline PORTAL DE TRANSPARENCIA & $\mathrm{I}$ \\
\hline INFORMES EXTERNOS AUDITORÍA & $\mathrm{I}$ \\
\hline ENTREGA CUENTAS ANUALES AL CSD & $\mathrm{I}$ \\
\hline FECHA DE ENTREGA EN EL REG. MERCANTIL & $\mathrm{I}$ \\
\hline ENVIO DE LAS CUENTAS A LA LIGA & $\mathrm{I}$ \\
\hline PUBLICACIÓN DE LOS ESTADOS FINANC. & $\mathrm{I}$ \\
\hline PUBLICACIÓN PUNTO DE EQUILIBRIO & $\mathrm{I}$ \\
\hline CUENTASANUALES DE ENT. VINCULADAS & $\mathrm{I}$ \\
\hline RATIO DE AUTONOMIIA FINANCIERA & $\mathrm{I}$ \\
\hline RETRIBUCIONES1ä PLANTILLA/PRESUPUESTO & 0 \\
\hline RATIO DEUDAS/INGRESOS RELEVANTES & $\mathrm{I}$ \\
\hline INVERSIÓN 1aPLANTILLA/ING. REL. (último año) & $\mathrm{I}$ \\
\hline DESGLOSE INGRESOS RELEVANTES & $\mathrm{I}$ \\
\hline DETALLE GASTOS RELEVANTES & $\mathrm{I}$ \\
\hline DETALLE GASTOS DE 1̇ PLANTILLA & $\mathrm{I}$ \\
\hline DESGLOSE DE ENDEUDAMIENTO & $\mathrm{I}$ \\
\hline LISTADEUDAS CON ADMIN. PÚBLICAS & $\mathrm{I}$ \\
\hline DEUDASY CRÉDITOSCON LOS EMPLEADOS & $\mathrm{I}$ \\
\hline DEUDASY CRÉDITOSCON EL PERSONAL DEP. & $\mathrm{I}$ \\
\hline IMPORTE DE TRASPASOS Y FICHAIES (2 últ años) & 0 \\
\hline TOPE SALARIAL DEL CLUB & 0 \\
\hline AYUDAS PÚBLICAS Y SUBVENCIONES RECIBIDAS & $\mathrm{I}$ \\
\hline PUBLICACIÓN DE LOS PRESUPUESTOS & $\mathrm{I}$ \\
\hline
\end{tabular}

CLUB J: (RESULTADO NO VÁLIDO). Se trata de un caso especial. Este club cuenta con un apartado de transparencia en el que se 
puede ver un enlace llamado textualmente «INFUT», en teoría un apartado en el que la entidad va a facilitar los datos de transparencia. Lo cierto es que la dirección a la que nos dirige el enlace es errónea, un hecho que no es anecdótico, ya que la anomalía se ha mantenido durante los últimos meses. Por ello, y pese a que en el apartado en sí el club facilita algún dato, se ha analizado este caso como erróneo.

Haciendo un análisis global de los resultados, se pueden distinguir claramente tres grupos: dos clubes con transparencia total, un equipo con una "falsa transparencia que no se ha podido analizar» y el resto, pese a que cada uno está definido por unas características concretas, tiene un carácter bastante homogéneo. En este análisis más general también hay que destacar la no presencia de un patrón respecto a los indicadores. Pese a que la mayoría de equipos contestan a un número similar de variables, no hay un conjunto de estas que se hayan olvidado (a escala general). Únicamente se debe hacer una excepción, la que se refiere al «importe por traspasos y fichajes de las dos últimas temporadas». Esta información solo la ofrecen tres clubes de los analizados (los dos totalmente transparentes, y el $\mathrm{G}$ ).

Un dato que tiene mucho que ver con la información teórica aportada al principio del estudio: no es casualidad que, de forma sistemática, los clubes no ofrezcan este dato en una época en la que la burbuja del fútbol parece haberse roto, precisamente, por el encarecimiento y el oscurantismo de las operaciones deportivas (compra y venta de jugadores).

\section{Conclusiones}

No cabe duda que la transparencia económica se ha erigido como un pilar fundamental en el ejercicio de los clubes de fútbol. Como hemos podido comprobar, en la actualidad, los principales equipos de LaLiga han incrementado la presentación al público de información relacionada con sus ejercicios anuales y sus cuentas, pues todos ellos, hoy en día, superan la cantidad de datos que ofrecían hace apenas dos años en análisis similares al este. Es evidente que, aunque también responda a que tienen más conciencia de que la transparencia es indispensable para la legitimación de su actividad en el mercado, la nueva ley impulsada por el máximo organismo del fútbol mundial, la FIFA, puede entenderse como el motivo principal que explique esta irrupción de la transparencia económica en los clubes de fútbol.

Por ello, si bien se debe confirmar la hipótesis de que se ha agrandado la cantidad de publicaciones de datos en los principales clubes de LaLiga, se tiene también que destacar este precepto de la FIFA. Igualmente, tampoco se deben olvidar los recientes 
acontecimientos relacionados con la Federación Española de Fútbol y los supuestos indicios de corrupción ni los relacionados con flamantes fichajes de dudoso baremo económico, precisamente, por la falta de transparencia. Con esto se quiere resaltar la posición de tema de actualidad y de polémica que configura el concepto de la transparencia económica entre los aficionados al deporte rey.

En definitiva, esto manifiesta que más que la adopción de un aspecto con el que diferenciarse y ofrecer una imagen de cercanía y responsabilidad, los clubes de fútbol siguen viendo en la transparencia económica una cuestión meramente impuesta por los organismos que regulan su actividad y simplemente se limitan a cumplir, algunos mínimamente, con el mandato. Esto explica el caso $\mathrm{J}$ : aparentemente el apartado está disponible pero luego no hay información ni, por lo visto durante la elaboración de esta investigación, se la espera. Del mismo modo, los casos $\mathrm{H}$ y $\mathrm{E}$, en los que se limitan a escanear un documento y publicarlo, dejan ver a las claras la poca implicación que tiene el personal del club en facilitar el acceso a la información; todo lo contrario, se realiza el mínimo exigible: que aparezcan los datos, pese a que si se desea un dato concreto hay que recurrir a las más de doscientas páginas que componen el documento.

Por tanto, se puede confirmar otra de las hipótesis planteadas en el comienzo del análisis: aunque los datos económicos de cada club sí se encuentran al alcance del usuario en la página web oficial de cada uno, estos ocupan un lugar residual dentro de la estructura del portal digital, es decir, para dar con ellos se tienen que buscar a conciencia; en una navegación sin este propósito claro el visitante difícilmente accede a estos datos.

Por otro lado, los resultados arrojan una deducción que comporta refutar el planteamiento inicial en el que se vaticinaba una diferencia en la transparencia económica en función de la forma jurídica. Los diferentes niveles de transparencia de cada club no permiten establecer una diferenciación entre clubes deportivos y Sociedades Anónimas Deportivas desde este prisma, pues en ambos casos se pueden observar distintos grados de transparencia. $\mathrm{Ni}$ mucho menos la Sociedad Anónima Deportiva, pese a presentar mayores obligaciones en cuanto a la publicación de sus cuentas anuales, es un seguro de transparencia; esta variable no parece ser un factor determinante. Así, se pueden ver clubes deportivos con una máxima nota de transparencia en este estudio y Sociedades Anónimas Deportivas con la misma puntuación.

Sin embargo, es innegable que todos los clubes analizados han hecho un esfuerzo, mayor o menor, por aumentar su transparencia en materia económica, como demuestra la comparación entre los datos obtenidos en este caso y los extraídos del informe de INFUT de 2016: todos han aumentado su puntuación. 
No obstante, aunque los equipos objeto de estudio de esta investigación presenten la mayoría de los datos analizados, la falta de interés en facilitar el acceso a estos por parte de los clubes en los que se limitan a colgar en la página web un documento, incluso páginas escaneadas, sumada a las variables ausentes en los casos que no presentan una transparencia total, como es el propósito de la FIFA, demuestran que todavía queda mucho por avanzar en esta materia.

\section{Nuevas líneas para futuros trabajos}

- La principal línea abierta que se ha quedado una vez elaborada la investigación es la que se refiere al apartado formal en cuanto a lo comunicativo. Más allá de partir de la base de variables que se aportan o no se aportan (puramente informativas) profundizar en el cómo, es decir, el grado de accesibilidad que los clubes de fútbol presentan en lo que respecta a transparencia financiera.

- Comparar, tanto en la línea estrictamente informativa que se ha seguido en el estudio como en un futurible trabajo que evalúe el apartado formal, las diferencias que presentan los clubes de primera y de segunda división respecto a la transparencia financiera.

\section{Bibliografía}

Antezana J, Luis H. 2003. "Fútbol: espectáculo e identidad». En Futbologias: Futbol, identidad y violencia en America Latina, editado por Pablo Alabarces, 85-95. Buenos Aires: Consejo Latinoamericano de Ciencias Sociales.

Bromberger, Christian. 1990. "Ciuccio e fuochi d'artificio: indagine sul rapporto fra la squadra di calcio napoletana e la sua città». Micromega 4 (90): 57-93.

Bromberger, Christian. 2001. Fútbol. Significados de la pasión popular por los clubes de fútbol. Buenos Aires: Siruela

Estrada Valls, Alba, Lirios Flores Córdoba, Cristina García González y Miriam Salgado Cervera. 2012. «Análisis de la comunicación financiera online como herramienta para la contribución a la reputación empresarial». Fòrum de Recerca 17: 505-514.

Gay de Liébana, José María. 2016. La gran burbuja del fútbol: los modelos de negocio que oculta el deporte más importante del mundo. Barcelona: Penguin Random House Grupo Editorial, SAU. 
González, Rayco. 2011. «El espectador deportivo y la construcción de la identidad cultural». Nómadas. Revista Crítica de Ciencias Sociales y Jurídicas 29 (1): 27-40.

Gutiérrez Gilsanz, Andrés. 2001. «La conversión de clubes deportivos en Sociedades Anónimas Deportivas». Revista de derecho de sociedades 17: 179-198.

Pérez-Díaz, Víctor, y Joaquín P. López Novo. 2003. El tercer sector social en España. Madrid. Ministerio de Trabajo y Asuntos Sociales.

Piazzo, Virginia. 2012. Crisis en la Web 2.0: Gestión de la Comunicación para Preservar la Imagen y la Reputación Organizacional Online. Tesis doctoral. Universidad Autónoma de Barcelona.

Riesgo, Jonathan. 2016. Transparencia económica-financiera en los clubes de fútbol españoles. Oviedo: Universidad de Oviedo.

Transparency International España. 2015. Índice de transparencia de los clubes de fútbol (INFUT). Disponible en https://transparencia.org.es/infut-2015/. 